\title{
Distributed Modeling and Control of Large-Scale Highly Flexible Solar-Powered UAV
}

\author{
Rui Wang, ${ }^{1}$ Zhou Zhou, ${ }^{1}$ Xiaoping $\mathrm{Zhu}{ }^{2}$ and Xiaoping Xu${ }^{1}$ \\ ${ }^{1}$ School of Aeronautics, Northwestern Polytechnical University, Xian 710072, China \\ ${ }^{2}$ Institute of UAV, Northwestern Polytechnical University, Xian 710072, China \\ Correspondence should be addressed to Rui Wang; wangrui@nwpu.edu.cn
}

Received 16 January 2015; Revised 27 March 2015; Accepted 16 April 2015

Academic Editor: Jaromir Horacek

Copyright (c) 2015 Rui Wang et al. This is an open access article distributed under the Creative Commons Attribution License, which permits unrestricted use, distribution, and reproduction in any medium, provided the original work is properly cited.

\begin{abstract}
The modeling, stability, and control characteristics of a large scale highly flexible solar-powered UAV with distributed all-span multielevons were presented. A geometrically nonlinear intrinsic beam model was introduced to establish the structural/flight dynamics coupled equation of motion (EOM); based on it, the explicit decoupled linear flight dynamics and structural dynamics EOM were derived through mean axis theory. Undeformed, deformed, and flexible models were compared through trimming and modal analysis. Since the deformation of wing has increased the UAV's moment of inertia about the pitch axis, the frequency of short period mode has obviously decreased for the deformed model. The serious coupling between short period mode and 1st bending mode also significantly influences the roots of short period mode of flexible model. So flexible model was the only one which is able to accurately estimate the flight dynamics behaviors and was selected as the later control model. Forty distributed elevons and LQG/LTR controller were employed to control the attitude and suppress the aeroelastic deformation of the UAV simultaneously. The dynamics performance, robustness, and simulation results show that they were suitable for large scale highly flexible solar-powered UAV.
\end{abstract}

\section{Introduction}

The wing of high aspect-ratio solar-powered UAV is highly flexible and the surface density of structure is much lower than other kinds of aircraft, which may lead to the huge scale of solar-powered UAV in order to carry sufficient payload. These characteristics will lead to significant geometrically nonlinear aeroelastic deformation during flight and strong coupling between aeroelasticity and flight dynamics. If these issues have not been taken into full account, large error will occur. One of the key recommendations from the flight mishap investigation of the most famous solar-powered UAV "Helios" was to "develop more advanced, multidisciplinary (structures, aeroelastic, aerodynamics, atmospheric, materials, propulsion, controls, etc) "time-domain" analysis methods appropriate to highly flexible, "morphing" vehicles" [1].

In the modeling of "morphing" vehicles, fruitful works have been presented by Hodges, Cesnik, and their coworkers. The analysis method of Hodges et al. underlying NATASHA
(Nonlinear Aeroelastic Trim And Stability of HALE Aircraft), a computer program that is based on geometrically exact, fully intrinsic beam equations and a finite-state induced flow model [2-4]. The University of Michigan's Nonlinear Aeroelastic Simulation Toolbox (UM/NAST) is developed by Cesnik and his coworkers $[5,6]$, which includes nonlinear strain-based beam model, unsteady aerodynamics with simplified stall models, and a six-degrees-of-freedom flight dynamic formulation.

In the study of flight dynamics of highly flexible UAV, [2] found that the behavior is distinctly different from that of a rigid aircraft while taking into account the flexibility effects. Because the low-frequency modes of structure are completely coupled to the flight dynamics mode, the phugoid as well as the short period mode is affected by wing flexibility significantly. Reference [3] furthered the study, the paper presented a theoretical basis for the flight dynamic response estimation of a highly flexible flying wing, multiple engines, and multiple control surfaces are taken into account. The results show that the phugoid mode may become unstable during large 
dihedral angles caused by large mass of payload pod. The pair of complex-conjugate short period roots merges to become two real roots. This is because the deformed " $U$ "shape leads to an order-of-magnitude increase in the pitch moment of inertia and so there is corresponding decrease in the frequency. Thus, this highly flexible aircraft does not show a classical short-period mode in its deformed state. Similar studies by Raghavan and Patil [4] and Su and Cesnik [5] confirmed this result. All the references paid attention to the nonlinear model and the results analysis, but the modeling of linear dynamics was ignored.

In [7], an optimal Static Output Feedback controller was designed for flutter suppression and gust load alleviation. However, only an elevon on the wing tip was employed in the controller, and the performance was highly dependent on sensor selection and placement.

In $[8,9]$, dynamic inversion control laws were presented for the trajectory control for Very Flexible Aircraft (VFA). However, in the use of dynamic inversion, there is an assumption that the stability and control derivatives remain fairly constant during the flight. This is not the case with highly flexible UAV.

In [10], a reduced-order $\mathrm{H}_{\infty}$ controller was employed to control a high speed civil transport aircraft, and the results implied that the reduced-order $\mathrm{H}_{\infty}$ controller was robust in the presence of unstructured uncertainty arising due to the model reduction error. However, the reduced order controller is weakly robust when employed to control the full-order model with parametric uncertainty.

LQG/LTR is another popular robust control method. The primary objective of LQG/LTR is to recover the robustness of LQG, an excellent full-state feedback robust controller. The procedure of LQG/LTR method is as follows: firstly, design a target feedback loop, which satisfies desired frequency performance, such as disturbance rejection and uncertainty deviation of the unmodeled dynamics, and then design a model based compensator to recover the properties of the target feedback loop [11]. LQG/LTR has been used widely in flight control, but only a few references are concentrated on the highly flexible aircraft.

In [12], an adaptive LQG/LTR controller was employed to control a very flexible aircraft, but the dynamics model was greatly simplified to capture only the unstable phugoid mode, and there were only 3 traditional control effectors, throttle, elevator, and aileron, available for the controller.

The large scale highly flexible UAV discussed in this paper is shown in Figure 1. It is equipped with 8 propulsion system nacelles (including secondary batteries, electrical motor, and propeller) and 5 vertical tails. The span is $70 \mathrm{~m}$ and the mean aerodynamics chord is $2.44 \mathrm{~m}$. In order to enhance the control ability, trailing-edge elevon spans the entire wing. Similar to the Helios [1], the elevon is divided into 40 distributed parts to release the notable aeroelastic deformation caused by large scale highly flexible wing.

For the aeroelastic flight dynamics analysis of such a UAV, most papers ignored the aerodynamics force distribution characteristic along the span and assumed the center of gravity (CG), elastic center (EC), and aerodynamics center (AC) to be coincident for simplification. In this paper,

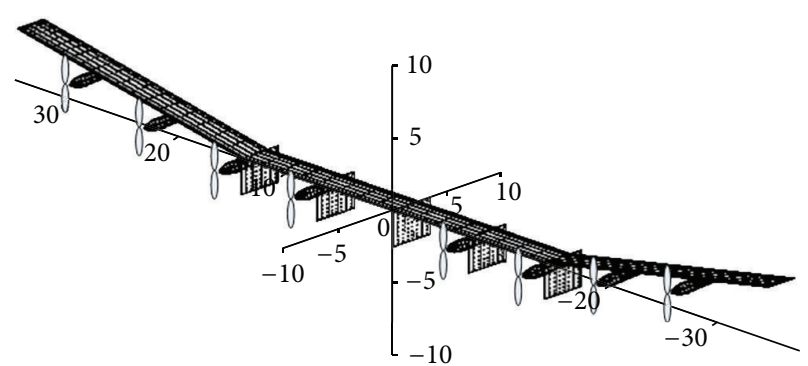

FIGURE 1: High aspect-ratio highly flexible solar-powered UAV.

TABLE 1: The mass parameters of high aspect-ratio solar-powered UAV.

\begin{tabular}{lccc}
\hline & $\begin{array}{c}\text { Coefficient of } \\
\text { mass }\end{array}$ & $\begin{array}{c}\text { CG (from } \\
\text { leading edge) }\end{array}$ & Elastic center \\
\hline $\begin{array}{l}\text { Structure } \\
\text { Solar array }\end{array}$ & 0.3 & $35 \%$ & $35 \%$ \\
$\begin{array}{l}\text { Propulsion } \\
\text { system }\end{array}$ & 0.15 & $45 \%$ & $/$ \\
\begin{tabular}{l} 
Payload \\
\hline
\end{tabular} & 0.5 & $0 \%$ & $/$ \\
& 0.05 & $\begin{array}{c}\text { (at the CG of } \\
\text { UAV) }\end{array}$ & $/$ \\
\hline
\end{tabular}

a more practical UAV model is proposed, in which the $\mathrm{AC}$ and $\mathrm{EC}$ is separated, the 3D quasisteady aerodynamics force is considered, and aerodynamics derivatives in every wing sections are derived for accuracy. In order to get a more accurate characteristic of solar-powered UAV, the mass parameters derived from conceptual design are employed, shown in Table 1.

In this paper, a nonlinear coupled structural and flight dynamics model based on geometrically nonlinear intrinsic beam was established first, and then its explicit linear dynamics model is derived for decoupled dynamics modal analysis by mean axis theory. At last, a LQG/LTR controller is designed with distributed 40 elevons for attitude control and aeroelastic deformation suppression for the large-scale highly flexible solar-powered UAV.

\section{Modeling of Nonlinear Structural/Flight Dynamics}

2.1. Structural Dynamics Model. The geometrically nonlinear structural dynamics and flight dynamics equations are based on the intrinsic beam equation derived by Hodges et al. shown as follows $[3,13]$ :

$$
\begin{aligned}
\dot{\bar{P}}+\widetilde{\bar{\Omega}} \bar{P} & =F^{\prime}+\widetilde{\bar{K}} \bar{F}+\bar{f} \\
\dot{\bar{H}}+\widetilde{\bar{\Omega}} \bar{H}+\widetilde{\bar{V}} \bar{P} & =M^{\prime}+\widetilde{\bar{K}} \bar{M}+\left(\tilde{e}_{2}+\widetilde{\bar{\gamma}}\right) \bar{F}+\bar{m} \\
\dot{\bar{\gamma}} & =V^{\prime}+\widetilde{\bar{K}} \bar{V}+\left(\widetilde{e}_{2}+\widetilde{\bar{\gamma}}\right) \bar{\Omega} \\
\dot{\bar{\kappa}} & =\Omega^{\prime}+\widetilde{\bar{K}} \bar{\Omega}
\end{aligned}
$$




$$
\begin{gathered}
\dot{\hat{P}}+\widetilde{\widehat{\Omega}} \widehat{P}=\widehat{f}, \\
\dot{\widehat{H}}+\widetilde{\widehat{\Omega}} \widehat{H}=\widehat{m} .
\end{gathered}
$$

The equations above are the core of structural dynamics, but they still cannot be used for the analysis of aerodynamics and flight dynamics without the relationship between displacements and rotations. Hence, the kinematics motion must be established to obtain the relationship between strain and displacement. The position vector of the origin of the deformed beam frame $(B-)$ in the root frame $(R-)$ and their rotational relationship are [14]:

$$
\begin{aligned}
\left\{r_{B}\right\}_{R} & =C_{R B}\left(\gamma+e_{2}\right), \\
C_{B R}^{\prime} & =-(\widetilde{\kappa}+\widetilde{k}) C_{B R} .
\end{aligned}
$$

The spatial derivatives can be replaced by the following equations, namely, central difference method [15]:

$$
\begin{aligned}
x_{i}^{\prime} & =\frac{1}{2 h}\left(x_{i+1}-x_{i-1}\right), \\
x_{i}^{\prime \prime} & =\frac{1}{h^{2}}\left(x_{i+1}-2 x_{i}+x_{i-1}\right), \\
x_{i}^{\prime \prime \prime} & =\frac{1}{2 h^{3}}\left(x_{i+2}-2 x_{i+1}+2 x_{i-1}-x_{i-2}\right), \\
x_{i}^{\prime \prime \prime \prime} & =\frac{1}{h^{4}}\left(x_{i+2}-4 x_{i+1}+6 x_{i}-4 x_{i-1}+x_{i-2}\right),
\end{aligned}
$$

where $h$ is the distance between two points.

The mean axis is applied to simplify and linearize the equation of motion. In the mean axis $\left(M_{-}\right)$frame, the linear and angular momentums due to elastic deformation are zero at every instant, which eliminates the coupling between the rigid body modes and the structural mode and results in a reduced-order system with only six degrees-of-freedom.

Since the equations in (1) are written in the $B$-frame, the equations of motion of rigid mode can be derived by premultiplying all terms by $C_{M B}$ to obtain components in the $M$ - frame and integrating in it along the beam axis as follows [16]:

$$
\begin{aligned}
& M_{\text {tot }}\left(\left\{\dot{V}_{c g}\right\}_{M}+\left\{\Omega_{M}\right\}_{M} \times\left\{V_{c g}\right\}_{M}\right)=\sum C_{M B_{i}}\left\{f_{n}\right\}_{B_{i}}, \\
& \left\{I_{c g}\right\}_{M}\left\{\dot{\Omega}_{M}\right\}_{M}+\left\{\dot{I}_{c g}\right\}_{M}\left\{\Omega_{M}\right\}_{M}+\left\{\Omega_{M}\right\}_{M} \\
& \quad \times\left(\left\{I_{c g}\right\}_{M}\left\{\Omega_{M}\right\}_{M}\right)=\sum\{r\}_{M} \times C_{M B_{i}}\left\{f_{n}\right\}_{B_{i}} \\
& \quad+\sum C_{M B_{i}}\left\{m_{i}\right\}_{B_{i}}-\sum C_{M B_{n}}\left\{I_{i}\right\}_{B}\left\{\dot{\Omega}_{i}\right\}_{B} \\
& \quad-\sum \dot{C}_{M B_{n}}\left\{I_{i}\right\}_{B}\left\{\Omega_{i}\right\}_{B},
\end{aligned}
$$

where the linear and angular velocities are defined as

$$
\begin{aligned}
& \left\{V_{c g}\right\}_{M}=\frac{1}{M_{\text {tot }}} \sum \mu_{i} C_{M B_{i}} V_{i}, \\
& \left\{\Omega_{M}\right\}_{M}=\left\{I_{c g}\right\}_{M}^{-1} \sum\left\{r_{B_{i} M}\right\}_{M} \times \mu_{i} C_{M B_{i}} V_{i} .
\end{aligned}
$$

The relationship of coordinate transformation is

$$
C_{M B}=C_{M g} C_{g B} .
$$

The total moment of inertia of the UAV is solved as follows:

$$
\left\{I_{c g}\right\}_{M}=\sum\left[C_{M B_{i}} I_{i} C_{M B_{i}}^{T}+\left(r_{i}^{T} r_{i} E-r_{i} r_{i}^{T}\right) m_{i}\right] .
$$

If the relative position of the rigid body does not vary significantly, $\left\{\dot{I}_{c g}\right\}_{M}$ can be approximated to be zero. If the motion of the UAV is limited to longitudinal only, the last two terms at the right hand of (5) can also be equal to zero.

\subsection{Force and Moment Model}

2.2.1. Internal Forces and Moments Model. The wing of solarpowered UAV may undergo large deformations during normal flight, while the strain is small, exhibiting geometrically nonlinear behaviors. Therefore, the small-strain assumption is suitable for this problem. The internal forces and moments are linearly related to the strains and curvatures, shown in (9) [17]:

$$
\left[\begin{array}{l}
F \\
M
\end{array}\right]=\left[\begin{array}{cc}
R & S \\
S^{T} & T
\end{array}\right]^{-1}\left[\begin{array}{l}
\gamma \\
\kappa
\end{array}\right] .
$$

The wing is assumed to be a prismatic and isotropic beam with the shear center and tension axis coincident with the reference axis, by which the above equation can be derived as follows:

$$
\begin{aligned}
& {\left[\begin{array}{c}
F_{x} \\
F_{y} \\
F_{z}
\end{array}\right]=\left[\begin{array}{lll}
G A_{x} & & \\
& E A & \\
& & G A_{z}
\end{array}\right]\left[\begin{array}{l}
\gamma_{x} \\
\gamma_{y} \\
\gamma_{z}
\end{array}\right],} \\
& {\left[\begin{array}{l}
M_{x} \\
M_{y} \\
M_{z}
\end{array}\right]=\left[\begin{array}{lll}
E I_{x} & \\
& G J & \\
& & E I_{z}
\end{array}\right]\left[\begin{array}{l}
\kappa_{x} \\
\kappa_{y} \\
\kappa_{z}
\end{array}\right],}
\end{aligned}
$$

where $G$ represents the shear modulus; $E$ represents Young's modulus; $J$ represents the torsional constant; $I_{x}$ and $I_{z}$ represent the area moment of inertia along the $x$ and $z$ direction.

2.2.2. Aerodynamics Model. In order to analyze the motion of the flexible UAV exactly, the aerodynamic force of vibratingwings should be analyzed. Unsteady aerodynamics models that are useful for aeroelastic analysis are usually Theodorsen unsteady aerodynamic model and finite state induced flow model of Peters and Cao [18]. The Theodorsen model is derived in frequency domain, but it can be converted into time domain by rational function approximation. The finite state induced flow model is derived in time domain, and it can be used in a state-space model easily. These two types of aerodynamics are equivalent and can be derived from one another through Laplace and Fourier transforms.

In this paper, the Theodorsen unsteady aerodynamics model is adopted, which has been used widely for airfoil or 
large aspect ratio wings, and its quasisteady form is relative to the traditional aerodynamics derivatives used in rigid aircraft.

For the Theodorsen model, the wing section is described in Figure 2, where the definitions of points are as follows: LE: leading edge, $G$ : center of gravity of the wing section, $g$ : center of gravity of the UAV, $A$ : aerodynamics center, $M$ : middle point of chord, $E$ : elastic center, the position in $x$ direction of $s$-frame is $\bar{a} b, C$ : control point of unsteady aerodynamics, and $D$ : aerodynamics center of control surface.

The definitions of variables are as follows: $\alpha$ : angle of attack, $b$ : length of $1 / 2$ chord, $u$ : deformation defined in $B$ frame, and $s$-frame: the origin is at LE, $x$ along the chord and pointing to the trailing edge, $z$ perpendicular to $x$ and point up.

The formulation of Theodorsen unsteady aerodynamics force and its moment to the elastic center are

$$
\begin{aligned}
C_{L} & =\pi b\left(\frac{\dot{\alpha}}{V}+\frac{\ddot{h}}{V^{2}}-\frac{b \bar{a} \ddot{\alpha}}{V^{2}}\right)+2 \pi C(k)\left(\frac{\dot{h}}{V}+\alpha\right. \\
& \left.+b\left(\frac{1}{2}-\bar{a}\right) \frac{\dot{\alpha}}{V}\right), \\
C_{M, E} & =\frac{\pi}{2}\left(\frac{b \bar{a} \hat{h}}{V^{2}}-\frac{b^{2}}{V^{2}}\left(\frac{1}{8}+\bar{a}^{2}\right) \ddot{\alpha}-\left(\frac{1}{2}-\bar{a}\right) \frac{b \dot{\alpha}}{V}\right) \\
& +\pi\left(\bar{a}+\frac{1}{2}\right) C(k)\left(\frac{\dot{h}}{V}+\alpha+b\left(\frac{1}{2}-\bar{a}\right) \frac{\dot{\alpha}}{V}\right) \\
& =\pi b\left(\frac{\dot{\alpha}}{V}\left[-\left(\frac{1}{4}-\frac{1}{2} \bar{a}\right)\right]+\frac{\ddot{h}}{V^{2}}\left[\frac{1}{2} \bar{a}\right]\right. \\
& \left.-\frac{b \bar{a} \ddot{\alpha}}{V^{2}}\left[\left(\frac{1}{8}+\bar{a}^{2}\right) \frac{1}{2} b\right]\right)+\left[\left(\frac{1}{4}+\frac{1}{2} \bar{a}\right)\right] 2 \pi C(k) \\
& +\left(\frac{\dot{h}}{V}+\alpha+b\left(\frac{1}{2}-\bar{a}\right) \frac{\dot{\alpha}}{V}\right),
\end{aligned}
$$

where $2 \pi$ is the theoretic lift slope of airfoil, the steady aerodynamics center is at $1 / 4$ chord and unsteady aerodynamics center and its control point is at $3 / 4$ chord. All these variables can be replaced by the actual airfoil data. $C(k)$ is the Theodorsen function, produced by circulation. $k=\omega b / V$ is the reduced frequency. If $k$ is low enough and $C(k)=0$, the flow will become quasisteady. As the flight dynamics and control of solar-powered UAV on low frequency is more concerned, quasisteady flow assumption is adopted for analysis in this paper.

By applying the quasisteady flow assumption and ignoring the terms in high orders, (11) becomes

$$
\begin{aligned}
C_{L}= & C_{L \alpha}\left(\alpha+\frac{\dot{u}_{z}}{V}+(1-\bar{a}) \frac{b \dot{\alpha}}{V}\right), \\
C_{M, E}= & C_{L \alpha}\left(\frac{1}{4}+\frac{1}{2} \bar{a}\right)\left(\alpha+\frac{\dot{u}_{z}}{V}\right) \\
& +C_{L \alpha}\left(\frac{1}{4} \bar{a}-\frac{1}{2} \bar{a}^{2}\right) \frac{b \dot{\alpha}}{V},
\end{aligned}
$$

where the airfoil data have been replaced by the actual ones.

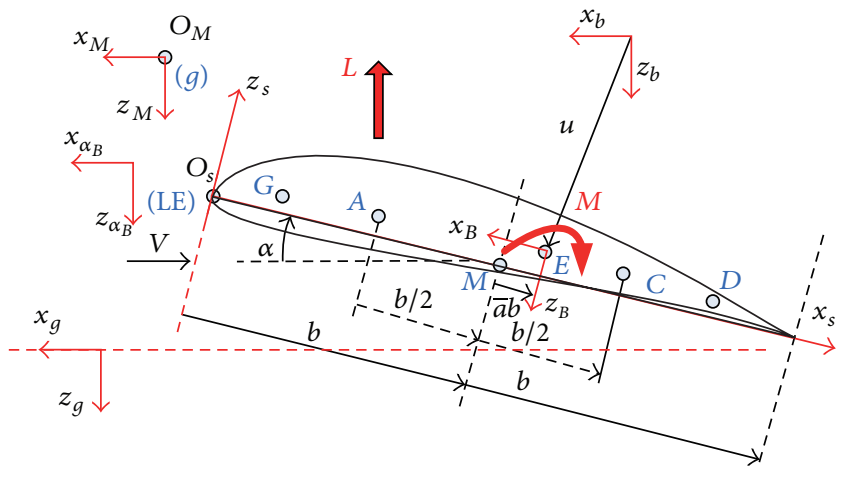

FIGURE 2: Illustration of wing section, coordinate systems, and variables.

The physical meaning of the above equations is that the lift of wing section is produced by steady and quasisteady flow together, and the steady part is produced by the angle of attack $\alpha$ of the section and its increment $\dot{u}_{z} / V_{0}$ caused by relative elastic deformation. The quasisteady part is caused by $\dot{\alpha}$.

It should be noted that, in most cases, the angles of attack in every sections (denoted as $\alpha_{B}$ ) are not easy to be measured directly. Hence, the relationship between $\alpha_{B}$ and incident angle at the CG of UAV expressed in $a_{M}$-frame (notated as $\alpha_{M}$ and $\beta_{M}$ ) can be calculated as

$$
\begin{aligned}
- & \sin \alpha_{B}=C_{a_{B} B}(3,1)=[0,0,1] \\
& \cdot C_{a_{B} a_{M}} C_{a_{M} M} C_{M B}[1,0,0]^{T} \\
& =\left(\cos \alpha_{M} \sin \beta_{M} \sin \varphi_{B M, x}-\sin \alpha_{M} \cos \varphi_{B M, x}\right) \\
& \cdot\left(\cos \varphi_{B M, y} \cos \varphi_{B M, z}\right)+\left(\cos \beta_{M} \sin \varphi_{B M, x}\right) \\
& \cdot\left(\cos \varphi_{B M, y} \sin \varphi_{B M, z}\right) \\
& -\left(\sin \alpha_{M} \sin \beta_{M} \sin \varphi_{B M, x}+\cos \alpha_{M} \cos \varphi_{B M, x}\right) \\
& \cdot \sin \varphi_{B M, y},
\end{aligned}
$$

where $\varphi_{B M}$ is the Euler angle from $B$-frame to $M$-frame and the subscripts $x, y$, and $z$ represent the 3 components of Euler angle, which correspond to the roll, pitch, and yaw angle of the aircraft, respectively.

If the wing rotates along the $x$ axis only, (13) can be simplified as

$$
\begin{aligned}
\sin \alpha_{B}= & -\cos \alpha_{M} \sin \beta_{M} \sin \varphi_{B M, x} \\
& +\sin \alpha_{M} \cos \varphi_{B M, x} .
\end{aligned}
$$

The magnitude of drag is small, and its influence on the flight dynamics characteristics is limited, so the drag on every wing section can be divided from the total drag. The expression of total drag is

$$
C_{D}=C_{D 0}+a_{\text {induce }} C_{L}^{2} .
$$


The aerodynamics force expressed in $a_{M}$-frame can be derived by the following axis conversion:

$$
\left\{f_{a}\right\}_{a_{M}}=C_{a_{M} a_{B}}\left\{f_{a}\right\}_{a_{B}}
$$

where

$$
C_{a_{M} a_{B}}=\left[\begin{array}{ccc}
1 & 0 & 0 \\
0 & \cos \varphi_{B M, x} & -\sin \varphi_{B M, x} \\
0 & \sin \varphi_{B M, x} & \cos \varphi_{B M, x}
\end{array}\right] .
$$

Substituting (17) into (16), the nondimensional force coefficients at the $a_{M}$-coordinate are derived as follows:

$$
\begin{aligned}
& \left\{C_{D}\right\}_{a_{M}}=\left\{C_{D}\right\}_{a_{B}}, \\
& \left\{C_{C}\right\}_{a_{M}}=\left\{C_{L}\right\}_{a_{B}} \sin \varphi_{B M, x}, \\
& \left\{C_{L}\right\}_{a_{M}}=\left\{C_{L}\right\}_{a_{B}} \cos \varphi_{B M, x} .
\end{aligned}
$$

Changing the reference point of moment into CG, the aerodynamics moment to CG of UAV is

$$
\left\{m_{a}\right\}_{M}=\left\{m_{a, 0}\right\}_{M}+\{r\}_{M} \times\left(C_{M a_{M}}\left\{f_{a}\right\}_{a_{M}}\right) .
$$

It should be noted that the method proposed in this section can be used to calculate either the aerodynamics force or the moment of vertical tails, although the incident angle and Euler angle may vary significantly.

2.2.3. 3D Effect Correction of Aerodynamics. In the analysis of the aeroelastic flight dynamics of solar-powered UAV, most papers ignored the aerodynamics force distribution characteristics along the wing span and considered the flow condition to be same as the airfoils. Actually, the wing span is finite, which will make the flow of each section affect each other; that is to say, $3 \mathrm{D}$ effect must be considered for precision. In this paper, 3D effect is corrected based on liftingline theory, and it applies well to wings that have aspect ratio greater than 5 and sweep angle less than 20 degrees.

According to lifting-line theory, the lift of airfoil is determined by effective angle of attack $\alpha_{\text {eff }}$, which is formulated as follows:

$$
\alpha_{\text {eff }}=\alpha_{\text {geo }}-\alpha_{\text {ind }}
$$

where $\alpha_{\text {geo }}$ and $\alpha_{\text {ind }}$ represent geometric and induced angle of attack, respectively.

According to the relationship of trailing vortex and circulation, the equation above becomes

$$
\begin{aligned}
\alpha_{\text {geo }}\left(y_{0}\right)= & \frac{\Gamma\left(y_{0}\right)}{\pi V_{\infty} c\left(y_{0}\right)}+\alpha_{L=0} \\
& +\frac{1}{4 \pi V_{\infty}} \int_{0}^{l} \frac{(d \Gamma / d y) d y}{y_{0}-y},
\end{aligned}
$$

where $y_{0}$ is an arbitrary spanwise position on the wing and $2 \pi$ refers to theoretical lift slope of airfoil, which can be substituted by true airfoil value in the actual computation. Since
(21) is a nonlinear integral-differential equation, coordinate transformation method should be applied before solving it for simplification [19].

After solving the circulation distribution $\Gamma(y)$ from (21), the lift distribution can be obtained from the KuttaJoukowski theorem:

$$
L\left(y_{0}\right)=\rho V_{\infty} \Gamma\left(y_{0}\right) .
$$

By repeating the above steps for different geometric angles of attack and taking a derivative, one can obtain the lift slope of each cross section of the wing considering 3D effect correction.

\section{Model Linearization}

3.1. Linearized Aerodynamics. The movement of wing section is separated into two parts: the rigid movement of UAV and the elastic movement of structure. Then, the lift coefficients applied on the wing section can be deployed in the $a_{B}$-frame as follows:

$$
\begin{gathered}
C_{L \alpha}, \\
C_{L q}=0.5 C_{L \alpha}+2 C_{L \alpha}\left(\frac{3}{4}-\left\{\bar{x}_{g}\right\}_{s}\right), \\
C_{L p}=-2 C_{L \alpha} \frac{\left\{y_{g}\right\}_{s}}{l}, \\
C_{L \varphi_{y}}=C_{L \alpha}, \\
C_{L \dot{u}_{z}}=C_{L \alpha} \frac{1}{V}, \\
C_{L \dot{\varphi}_{y}}=C_{L \alpha}(1-\bar{a}) \frac{c}{2 V} .
\end{gathered}
$$

It should be noted that the variables in the above equations are measured in the $a_{B}$-frame and $B$-frame.

According to the 2 nd equation of (12), the aerodynamics moment to the elastic center (EC) is

$$
\begin{aligned}
C_{m \alpha, E} & =-C_{L \alpha}\left\{\bar{x}_{A E}\right\}_{B} \approx C_{L \alpha}\left(\frac{1}{4}+\frac{1}{2} \bar{a}\right), \\
C_{m q, E} & =0.5 C_{L \alpha}\left\{\bar{x}_{C E}\right\}_{B}+2 C_{L \alpha}\left\{\bar{x}_{C g}\right\}_{s}\left\{\bar{x}_{A E}\right\}_{B}, \\
C_{m p, E} & =-2 C_{L \alpha} \frac{\left\{y_{g}\right\}_{s}}{l}\left\{\bar{x}_{A E}\right\}_{B}, \\
C_{m \varphi_{y}, E} & =C_{m \alpha, E}, \\
C_{m \dot{u}_{z}, E} & =C_{m \alpha, E} \frac{1}{V}, \\
C_{m \dot{\varphi}_{y}, E} & =C_{L \alpha}\left(\frac{1}{4} \bar{a}-\frac{1}{2} \bar{a}^{2}\right) \frac{c}{2 V} .
\end{aligned}
$$

From the 2nd equation of (18), the difference of lift expressed in the $a_{M}$-frame and $a_{B}$-frame is only $\cos \varphi_{B M, x}$. Hence, by replacing $C_{L \alpha}$ in (23) with $C_{L \alpha} \cos \varphi_{B M, x}$, the lift expressed in the $a_{B}$-frame will be derived. 
Substituting the expression of lift at $a_{M}$-frame into (19), the pitch moment about the center of gravity of the UAV can be obtained as follows:

$$
\begin{aligned}
C_{m \alpha, g} & =-C_{L \alpha} \cos \varphi_{M B, x}\left(\left\{\bar{x}_{A}\right\}_{s}-\left\{\bar{x}_{g}\right\}_{s}\right), \\
C_{m q, g} & =C_{L \alpha} \cos \varphi_{M B, x}\left(0.5\left\{\bar{x}_{C g}\right\}_{B}-2\left\{\bar{x}_{C g}\right\}_{B}^{2}\right), \\
C_{m p, g} & =-2 C_{L \alpha} \frac{\left\{y_{g}\right\}_{s}}{l}\left\{\bar{x}_{C g}\right\}_{B}, \\
C_{m \varphi_{y}, g} & =C_{m \alpha, g}, \\
C_{m \dot{u}_{z}, g} & =C_{m \alpha, g} \frac{1}{V} \\
C_{m \dot{\varphi}_{y}, g} & =-2 C_{L \alpha} \cos \varphi_{M B, x}\left(\frac{1}{2}-\left\{\bar{x}_{g}\right\}_{s}\right)^{2} \frac{b}{V}
\end{aligned}
$$

3.2. Linearized Flight Dynamics Equation. It can be found that the form of (4) and (5) is similar to that of flight dynamics equations of normal rigid vehicles. Hence, they can also be linearized by small disturbance method and written into two sets of equations: longitudinal and lateral-directional. Different from the normal rigid vehicle, the external force such as aerodynamics, thrust, and gravity are related to rigid motion and elastic motion for elastic model. The linear equation of flight dynamics for elastic UAV is

$$
\dot{\mathbf{x}}_{r}=\mathbf{A}_{r} \mathbf{x}_{r}+\mathbf{B}_{r} \mathbf{u}_{r}+\mathbf{A}_{r e} \mathbf{x}_{e}
$$

where $\mathbf{x}_{r}$ is the independent variable of rigid motion, with $\mathbf{x}_{r}=[v, \alpha, q, \theta]^{T}$ in longitudinal equations; $\mathbf{x}_{e}=$ $\left[\dot{u}_{x}{ }^{T}, \dot{u}_{z}{ }^{T}, \dot{\varphi}_{y}{ }^{T}, u_{x}{ }^{T}, u_{z}{ }^{T}, \varphi_{y}{ }^{T}\right]^{T}$ is the independent variable of elastic motion; $\mathbf{u}_{r}$ is the control variable of rigid motion, $\mathbf{u}_{r}=$ $\left[\delta_{e, 1} \cdots \delta_{e, 40}, \delta_{t}\right]^{T}$; that is, all the elevons can be controlled independently, but all throttles act conformably again for simplification; $\mathbf{A}_{r}$ is the same as conventional rigid vehicles as shown in [20]; and $\mathbf{B}_{r}$ is similar to the rigid model also, but the column is equal to the length of $\mathbf{u}_{r} ; \mathbf{A}_{r e}$ is the influence matrix of elastic motion to rigid motion, for longitudinal motion:

$$
\mathbf{A}_{r e}=\left[\begin{array}{cccccc}
0_{1 \times n} & 0_{1 \times n} & 0_{1 \times n} & 0_{1 \times n} & 0_{1 \times n} & 0_{1 \times n} \\
0_{1 \times n} & Z_{\dot{u}_{z}, 1} \cdots Z_{\dot{u}_{z}, n} & Z_{\dot{\varphi}_{y}, 1} \cdots Z_{\dot{\varphi}_{y}, n} & 0_{1 \times n} & 0_{1 \times n} & Z_{\varphi_{y}, 1} \cdots Z_{\varphi_{y}, n} \\
0_{1 \times n} & M_{\dot{u}_{z}, 1} \cdots M_{\dot{u}_{z}, n} & M_{\dot{\varphi}_{y}, 1} \cdots M_{\dot{\varphi}_{y}, n} & 0_{1 \times n} & 0_{1 \times n} & M_{\varphi_{y}, 1} \cdots M_{\varphi_{y}, n} \\
0_{1 \times n} & 0_{1 \times n} & 0_{1 \times n} & 0_{1 \times n} & 0_{1 \times n} & 0_{1 \times n}
\end{array}\right] .
$$

The details of $\mathbf{A}_{r e}$ can be found in [21].

3.3. Linearized Structural Dynamics Equation. Ignoring nonlinear coupling terms in (1), and assuming that the translational strain is small and the external moment and the moment of inertia of $x$ and $z$ direction are equal to zero, (1) can be reduced into

$$
\begin{aligned}
\mu \dot{V}_{x} & =E I_{z} \kappa_{z}^{\prime \prime}+f_{x}, \\
\mu \dot{V}_{z} & =-E I_{x} \kappa_{x}^{\prime \prime}+f_{z}, \\
I_{y y} \dot{\Omega}_{y} & =E I_{y} \kappa_{y}^{\prime}+m_{z}, \\
\dot{\kappa}_{x} & =V_{z}^{\prime \prime}, \\
\dot{\kappa}_{y} & =\Omega_{y}^{\prime}, \\
\dot{\kappa}_{z} & =-V_{x}^{\prime}, \\
\mu \dot{V}_{y} & =f_{y} .
\end{aligned}
$$

The equations above can be rearranged into a compact form as follows:

$$
\begin{aligned}
& \mu \ddot{x}+E I_{z} x^{\prime \prime \prime \prime}=f_{x}, \\
& \mu \ddot{z}+E I_{x} z^{\prime \prime \prime \prime}=f_{z},
\end{aligned}
$$

$$
\begin{aligned}
I_{y y} \ddot{\varphi}_{B g, y}-G J_{z} \varphi_{B g, y}^{\prime \prime} & =m_{y}, \\
\mu \dot{V}_{y} & =f_{y} .
\end{aligned}
$$

Since the focus of this paper is the flight dynamics characteristics of the total UAV, the structural dynamics characteristics are used to derive the increment of aerodynamics force caused by aeroelasticity only and many assumptions can be introduced to linearize the equations above for simplification.

Equation (29) can be simplified to the form as in (30) by rewriting in $M$-frame and assuming that the structural elastic vibrations are small perturbations near the static trim state and the elastic motion in $x$ direction is ignorable. In the case of geometry, it is assumed that the $M$-frame is parallel to $R$ frame and the sweep angle of wing is zero. Besides, in mean axis system, the elastic motion is influenced by rigid motion through aerodynamics force only:

$$
\begin{aligned}
\mu\left\{\ddot{u}_{x}\right\}_{M, x}+E I_{z}\left\{u_{x}\right\}_{M, x}^{\prime \prime \prime \prime} & =\cos \varphi_{B M, z}\{f\}_{B, x}, \\
\mu\left\{\ddot{u}_{z}\right\}_{M, z}+E I_{x}\left\{u_{z}\right\}_{M, z}^{\prime \prime \prime \prime} & =\cos \varphi_{B M, x}\{f\}_{B, z}, \\
I_{y y} \ddot{\varphi}_{B M, y}-G J_{z} \varphi_{B M, y}^{\prime \prime} & =\{m\}_{B, y},
\end{aligned}
$$

where $\varphi_{B M, y}$ represents the angle between $x$-axis of $B$-frame and the oxy plane of $M$-frame. All variables in (30) are in incremental form and the notation $\Delta$ has been neglected for simplification. 
Replacing the spatial derivatives by (3), and knowing the fact that the wing tip is free, the boundary conditions can be defined that the bending moments and shear forces at the first and last nodes are zero for the 2 nd equation in (30); that is [15],

$$
\begin{aligned}
& u_{z, 1}^{\prime \prime}=0, \\
& u_{z, n}^{\prime \prime}=0, \\
& u_{z, 1}^{\prime \prime \prime}=0, \\
& u_{z, n}^{\prime \prime \prime}=0 .
\end{aligned}
$$

Thus, the structural dynamics equation can be derived as follows:

$$
M_{u_{z}} \ddot{u}_{z}+K_{u_{z}} u_{z}=Q_{u_{z}}
$$

where

$$
\begin{aligned}
& u_{z}=\left[\begin{array}{lll}
u_{1} & \cdots & u_{n}
\end{array}\right]^{T} \text {, } \\
& M_{u_{z}}=\left[\begin{array}{lll}
\mu_{1} & & \\
& \ddots & \\
& & \mu_{n}
\end{array}\right] \text {, } \\
& K_{u_{z}}=\left[\begin{array}{ccccccc}
2 & -4 & 2 & & & & \\
-2 & 5 & -4 & 1 & & & \\
1 & -4 & 6 & -4 & 1 & & \\
& \ddots & \ddots & \ddots & \ddots & \ddots & \\
& & 1 & -4 & 6 & -4 & 1 \\
& & & 1 & -4 & 5 & -2 \\
& & & & 2 & -4 & 2
\end{array}\right] \\
& \text { * } \frac{1}{\Delta l^{4}} \operatorname{diag}\left(E I_{z, i}\right) \text {. }
\end{aligned}
$$

For the 2 nd equation in (30), it is the same as (33); that is,

$$
M_{u_{x}} \ddot{u}_{x}+K_{u_{x}} u_{x}=Q_{u_{x}} \text {. }
$$
[15]

For the 3rd equation in (30), the boundary conditions are

$$
\begin{aligned}
& \varphi_{B M, y, 1}^{\prime}=0, \\
& \varphi_{B M, y, n}^{\prime}=0 .
\end{aligned}
$$

Then, the structural dynamics equation becomes

$$
M_{\varphi_{y}} \ddot{\varphi}_{y}+K_{\varphi_{y}} \varphi_{y}=Q_{\varphi_{y}}
$$

where

$$
\begin{aligned}
\varphi_{y}= & {\left[\begin{array}{lll}
\varphi_{B M, y, 1} & \cdots & \varphi_{B M, y, n}
\end{array}\right]^{T}, } \\
M_{\varphi_{y}}= & {\left[\begin{array}{lll}
I_{1} & & \\
& \ddots & \\
& & I_{n}
\end{array}\right], } \\
K_{\varphi_{y}} & =-\left[\begin{array}{ccccc}
-2 & 2 & & \\
1 & -2 & 1 & \\
& \ddots & \ddots & \ddots & \\
& & 1 & -2 & 1 \\
& & & 2 & -2
\end{array}\right] * \frac{1}{\Delta l^{2}} \operatorname{diag}\left(G J_{i}\right) .
\end{aligned}
$$

In order to further simplify the linear structural dynamics equations, (33), (35), and (37) can be expanded in the modal coordinate system, and the final form can be derived as follows:

$$
\dot{\mathbf{x}}_{n e}=\mathbf{A}_{n e} \mathbf{x}_{n e}+\mathbf{A}_{n e r} \mathbf{x}_{r}+\mathbf{B}_{n e} \mathbf{u}_{r}
$$

where the independent variables are

$$
\begin{aligned}
\mathbf{x}_{n e} & =\left[\begin{array}{l}
\dot{\mathbf{x}}_{n} \\
\mathbf{x}_{n}
\end{array}\right], \\
\mathbf{x}_{e} & =\mu \mathbf{x}_{n}, \\
\mathbf{x}_{e} & =\left[u_{x}^{T}, u_{z}^{T}, \varphi_{y}^{T}\right]^{T},
\end{aligned}
$$

where $\mathbf{x}_{n}$ is the modal displacements of structural elastic motion, which is the symmetric modes for longitudinal motion; $\mu$ is the transfer matrix from modal coordinate to physical coordinate.

For longitudinal motion, the coefficient matrices in (39) are

$$
\begin{aligned}
& A_{n e}=\left[\begin{array}{cc}
M_{n}^{-1} Q_{n 1} & M_{n}^{-1}\left(Q_{n 2}-K_{n}\right) \\
I & 0
\end{array}\right], \\
& A_{n e r}=\left[\begin{array}{c}
M_{n}^{-1} Q_{n 0} \\
0
\end{array}\right], \\
& B_{n e}=\left[\begin{array}{c}
M_{n}^{-1} Q_{n 3} \\
0
\end{array}\right], \\
& M_{n}=\mu^{T} M \mu, \\
& K_{n}=\mu^{T} K \mu, \\
& Q_{n i}=\mu^{T} Q_{i},
\end{aligned}
$$




$$
\begin{aligned}
Q_{n 0} & =\mu^{T} Q_{0}, \\
Q_{n 1} & =\mu^{T} Q_{1} \mu, \\
Q_{n 2} & =\mu^{T} Q_{2} \mu, \\
Q_{n 3} & =\mu^{T} Q_{3} \mu, \\
Q_{0} & =\left[\begin{array}{cccc}
X_{v, 1} & X_{\alpha, 1} & & \\
\vdots & \vdots & 0_{n \times 1} & 0_{n \times 1} \\
X_{v, n} & X_{\alpha, n} & & \\
Z_{v, 1} & Z_{\alpha, 1} & Z_{q, 1} & \\
\vdots & \vdots & \vdots & 0_{n \times 1} \\
Z_{v, n} & Z_{\alpha, n} & Z_{q, n} & \\
M_{v, 1} & M_{\alpha, 1} & M_{q, 1} & \\
\vdots & \vdots & \vdots & 0_{n \times 1} \\
M_{v, n} & M_{\alpha, n} & M_{q, n} &
\end{array}\right],
\end{aligned}
$$

$Q_{1}$

$$
=\left[\begin{array}{ccc}
X_{\dot{u}_{x}, 1} \cdots X_{\dot{u}_{x}, n} & X_{\dot{u}_{z}, 1} \cdots X_{\dot{u}_{z}, n} & 0_{n \times 1} \\
Z_{\dot{u}_{x}, 1} \cdots Z_{\dot{u}_{x}, n} & Z_{\dot{u}_{z}, 1} \cdots Z_{\dot{u}_{z}, n} & Z_{\dot{\varphi}_{y}, 1} \cdots Z_{\dot{\varphi}_{y}, n} \\
0_{n \times 1} & M_{\dot{u}_{z}, 1} \cdots M_{\dot{u}_{z}, n} & M_{\dot{\varphi}_{y}, 1} \cdots M_{\dot{\varphi}_{y}, n}
\end{array}\right],
$$$$
Q_{2}=\left[\begin{array}{ccc}
0_{1 \times n} & 0_{1 \times n} & X_{\varphi_{y}, 1} \cdots X_{\varphi_{y}, n} \\
0_{1 \times n} & 0_{1 \times n} & Z_{\varphi_{y}, 1} \cdots Z_{\varphi_{y}, n} \\
0_{1 \times n} & 0_{1 \times n} & M_{\varphi_{y}, 1} \cdots M_{\varphi_{y}, n}
\end{array}\right] \text {, }
$$$$
Q_{3}=\left[\begin{array}{cccc}
X_{\delta_{e, 1}} & \cdots & X_{\delta_{e, n}} & X_{\delta_{t}} \\
Z_{\delta_{e, 1}} & \cdots & Z_{\delta_{e, n}} & Z_{\delta_{t}} \\
M_{\delta_{e, 1}} & \cdots & M_{\delta_{e, n}} & M_{\delta_{t}}
\end{array}\right]
$$

where $Z_{\dot{u}_{z}}$ is related to some derivatives (e.g., $C_{L \dot{u}_{z}}$ ). The exact expressions are the same as the derivatives of general flight dynamics equations. More details about them can be referred to $[21]$.

3.4. Total Linearized Equation. Combining (26) and (39), the final total linear equations can be derived as follows:

$$
\begin{gathered}
\dot{\mathbf{x}}_{r}=\mathbf{A}_{r} \mathbf{x}_{r}+\mathbf{A}_{r n e} \mathbf{x}_{n e}+\mathbf{B}_{r} \mathbf{u}_{r}, \\
\dot{\mathbf{x}}_{n e}=\mathbf{A}_{n e r} \mathbf{x}_{r}+\mathbf{A}_{n e} \mathbf{x}_{n e}+\mathbf{B}_{n e} \mathbf{u}_{r} .
\end{gathered}
$$

The independent variable is

$$
\mathbf{x}=\left[\mathbf{x}_{r}^{T}, \mathbf{x}_{n e}^{T}\right]^{T}
$$

and the state matrix is

$$
A=\left[\begin{array}{cc}
A_{r} & A_{r n e} \\
A_{n e r} & A_{n e}
\end{array}\right] .
$$

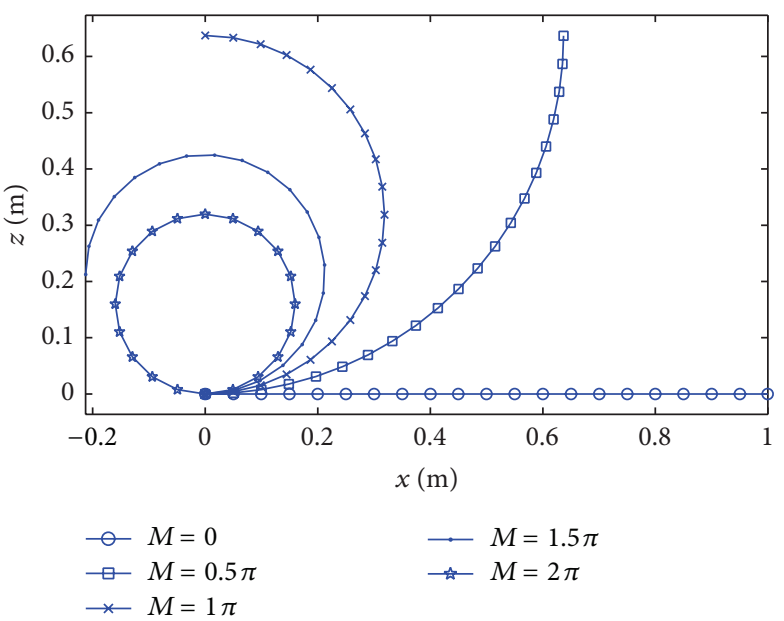

Figure 3: Beam deformation with increasing tip moment.

And the control matrix is:

$$
B=\left[\begin{array}{c}
B_{r} \\
B_{n e}
\end{array}\right] .
$$

The eigenvalues of the above state matrix are the modal eigenvalues of the elastic UAV considering the coupling of aeroelastic and flight dynamics:

$$
\dot{\mathbf{x}}=\mathbf{A x}+\mathbf{B u} .
$$

\section{Model Verification}

4.1. Geometrically Nonlinear Deformation. In order to validate the effectiveness of the geometrically nonlinear structural model and central difference method proposed in this paper, a cantilever beam with bending rigidity $E I=1$ and length $l=1 \mathrm{~m}$ is employed. While applying increasing moment $M$ at the tip of the beam, the deformation of the beam is shown in Figure 3. It can be seen that while $M=2 \pi$, the angular displacement is $2 \pi$, the tip coincides with the root and the beam is deformed into a circle, which is consistent with the analytical results [16]. The effectiveness of structural model is validated.

4.2. Aerodynamics Model. Aerodynamics model is the most complex and important external force model in the problem of flight dynamics and control, so it must be checked carefully before further studies. The lift coefficient distribution along the wing span obtained from two methods are compared in Figure 4; one is derived from the Thin-Strip lifting-line theory proposed in Section 3.1 in this paper, and the other is the Vortex-Lattice method from AVL software. It can be seen that the precision of Thin-Strip lifting-line theory model can satisfy the application requirements of this paper.

4.3. Linear Structural Model. In order to investigate the error of different modal truncation, 4 linear structural models of the wing are compared with the geometrically nonlinear 


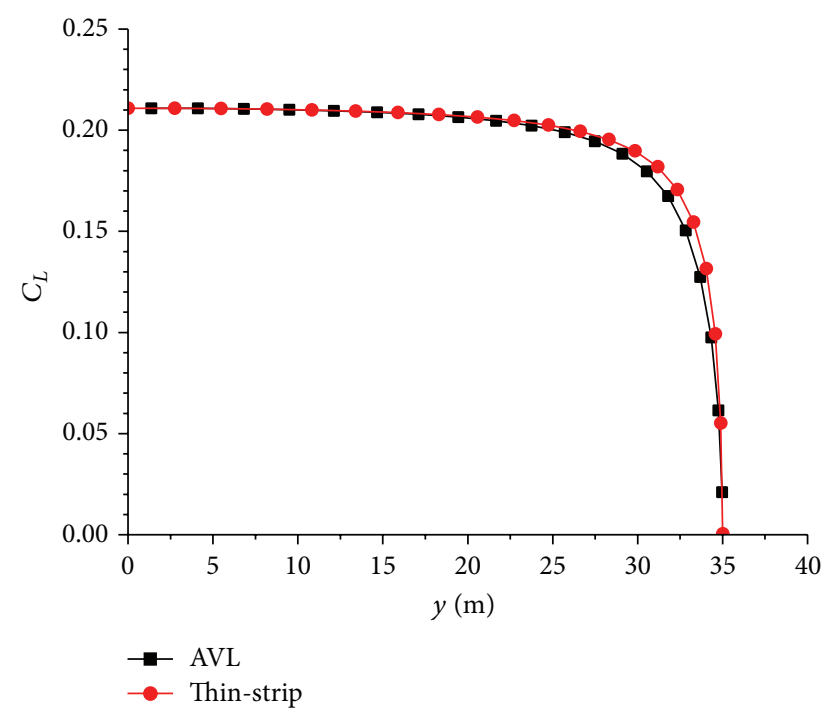

Figure 4: Comparison of spanwise lift coefficient distribution.

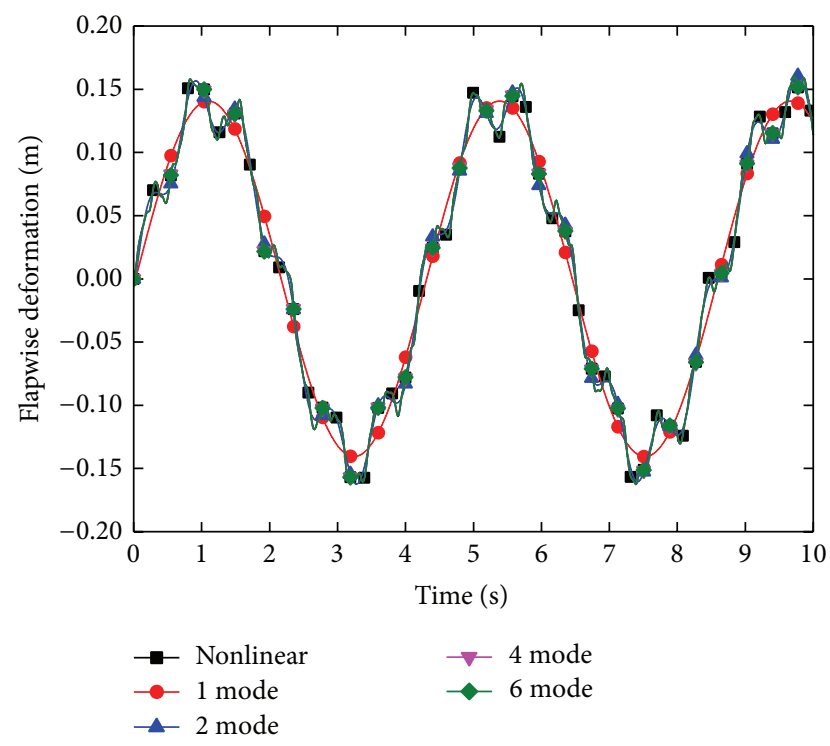

FIGURE 5: Time domain responses of the wing tip of different truncated linear wing models.

one: truncating at the 1st, 2nd, 4th, and 6th bending mode, respectively, and then applying a flapwise impulse force to the tip of the wing. The time domain responses of the wing tip of different models are shown in Figure 5.

It can be seen that, if the deformation is not large enough, for the flapwise deformation, if we employ the first 6 bending modes to constitute the linear structural model, the responses of the wing tip are coincident with the nonlinear one well. Similarly, it can be found from simulation that the first 4 twist modes and 2 chordwise modes are precise enough. So, the first 6 flapwise bending, 4 twist and 2 chordwise bending modes are adopted to constitute the linear structural model of the wing in the following of this paper.

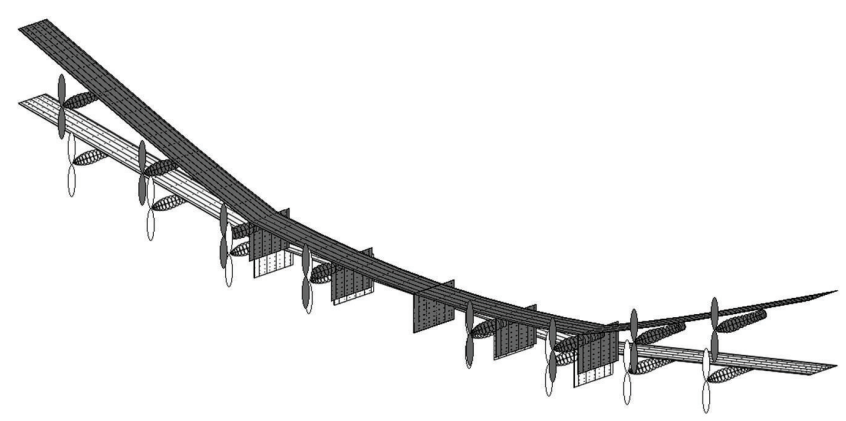

FIgURE 6: Deformed UAV in trimming.

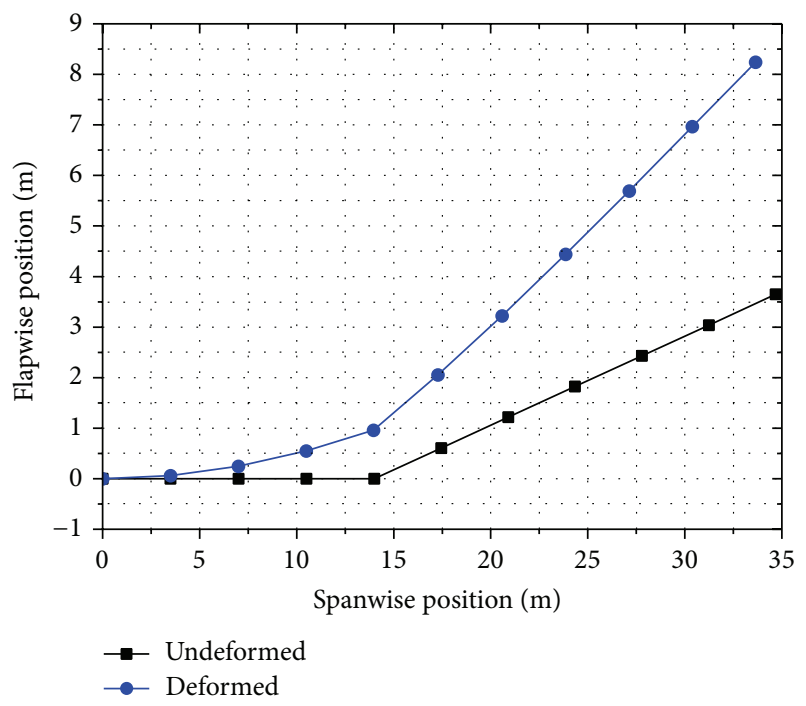

FIGURE 7: Flapwise position of UAV of different calculation model.

\section{Flight Dynamics of Highly Flexible UAV}

5.1. Trimming. Two kinetics models are employed for trimming: the "Undeformed" model represents the common rigid flight dynamics model; the "Deformed" model corresponding to (1) represents the flexible flight dynamics model.

In level flight, assuming that all the 40 distributed elevons deflect conformably for trimming for simplification, the highly flexible UAV will deform under the aeroelastic effect. In fact, the deformed UAV in trimming is shown in Figure 6, are the flapwise position and pitch angle of the UAV are shown in Figures 7 and 8.

It can be seen that the maximum wing deformation is $4.58 \mathrm{~m}$ at the wing tip, and the pitch angle at wing tip is larger than the root for about $3^{\circ}$. Larger pitch angle brings larger lift, and larger lift at wing tip will bring larger deformation. So, all the distributed elevons deflecting conformably is not the optimal control case while considering aeroelasticity.

5.2. Modal Analysis. The "Underformed" and "Deformed" models are corresponding to conventional rigid vehicles, and the linear "Flexible" model is based on (42). The longitudinal modes of different calculation models are shown in Table 2. 


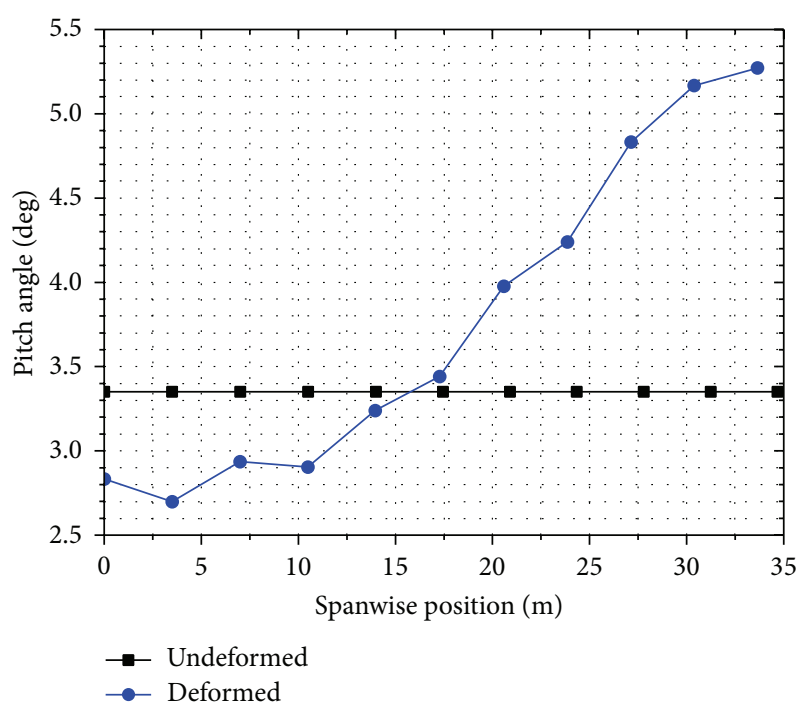

FIGURE 8: Pitch angle of UAV of different calculation model.

TABLE 2: Roots of longitudinal mode of different calculation models.

\begin{tabular}{lccc}
\hline & Short period & Phugoid & 1st bending \\
\hline Undeformed & $-6.710 \pm 3.925 i$ & $-0.046 \pm 1.003 i$ & $/$ \\
Deformed & $-5.985 \pm 3.100 i$ & $-0.002 \pm 1.063 i$ & $/$ \\
Flexible & $-0.394 \pm 1.707 i$ & $0.002 \pm 1.031 i$ & $-3.819 \pm 1.459 i$ \\
\hline
\end{tabular}

The results showed that the difference of these models is huge in longitudinal mode. Since the deformation of wing has increased the UAV's moment of inertia about the pitch axis, the frequency of short period mode has obviously decreased for the deformed model. The serious coupling between short period mode and 1st bending mode also significantly influences the roots of short period mode, which then results in the phugoid changing from stable to unstable. These tendencies are similar to the results presented by Hodges et al. Through these analyses, it is clear that the flexible model is the only one which is able to accurately estimate the longitudinal flight dynamics behaviors.

\section{Distributed LQG/LTR Controller Design}

6.1. Statement of LQG/LTR Control. For a standard state space system described in (46), the design procedure of LQG/LTR controller in the output port is as follows:

(1) Design a LQR regulator $F$, which ensures that the singular value of open loop system $F G(s)$ on a certain frequency satisfies the robustness requirement.

(2) Design a target loop: select appropriate $\Xi$ and $\Theta$, so as to the singular value of full-state feedback loop transfer function $G_{c}(s) G(s)$ on the considering frequency close to that of $F G(s)$ adequately.

Where, the controlled plant $G(s)$ is

$$
G(s)=C(s I-A)^{-1} B
$$

and the derived LQG/LTR controller plant $G_{c}(s)$ is

$$
G_{c}(s)=F(s I-A+B F+L C)^{-1} L .
$$

The methodology of LQR is searching a full state feedback controller $u=-F x$ to minimize the quadratic cost function:

$$
J=\min \left(\int_{0}^{\infty}\left(x^{T} Q x+u^{T} R u\right) d t\right)
$$

subject to the system dynamics described in (46).

The expression of $F$ can be calculated by

$$
F=R^{-1} B^{T} P
$$

where $P$ is the solution of the following algebraic Riccati equation:

$$
A^{T} P+P A-P B R^{-1} B^{T} P+Q=0 .
$$

The solution of $L$ in (48) is similar to $F$; one just needs to replace the matrix $A, B, Q, R$ in (49)-(51) to $A^{T}, C^{T}, \Xi+$ $\rho B B^{T}, \Theta$, respectively, where $\Xi+\rho B B^{T}$ represents the noise intensity; different noise intensity can be adjusted by $\rho$; $\Theta$ represents the sensor noise intensity. A well-designed LQG/LTR controller should be that, when $\rho \rightarrow \infty$, the singular value of $G_{c}(s) G(s)$ on the considering frequency close to that of $F G(s)$ adequately. But it should be noted that larger $\rho$ will derive larger gain of $G_{c}(s)$.

6.2. Designing LQG/LTR Pitch Angle Controller. Before designing LQG/LTR control law, one shall augment the plant to satisfy the specific performance requirement. For example, adding integrators will assure the zero steady-state errors to step inputs. So, in order to control the pitch angle precisely, the integrator of pitch angle $\theta_{i}$ and velocity $V_{i}$ should be included in the control plant. Then, the control plant in (46) becomes

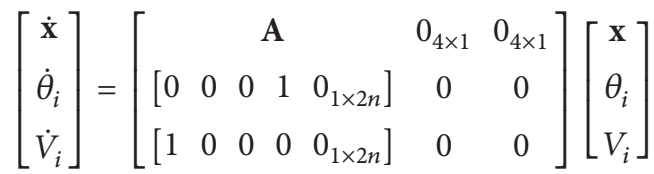

$$
\begin{aligned}
& +\left[\begin{array}{l}
\mathbf{B} \\
0 \\
0
\end{array}\right] \mathbf{u} \text {. }
\end{aligned}
$$

The critical factor for a practical controller is that not all the state variables are easy to be measured in engineering. So only a part of variables can be used to implement the output feedback control. Because the LTR technology can recover the stable margin of LQR, which is known as an excellent state feedback robust controller, the robustness of LQG/LTR output feedback controller can be guaranteed.

Because the flapwise bending motion of the wing is easy to be measured, so $u_{z}$ together with $V, \alpha, q, \theta, \theta_{i}, V_{i}$ are selected to be the measured variables for the controller. Then, we can apply the LQG/LTR method described above in (52). 


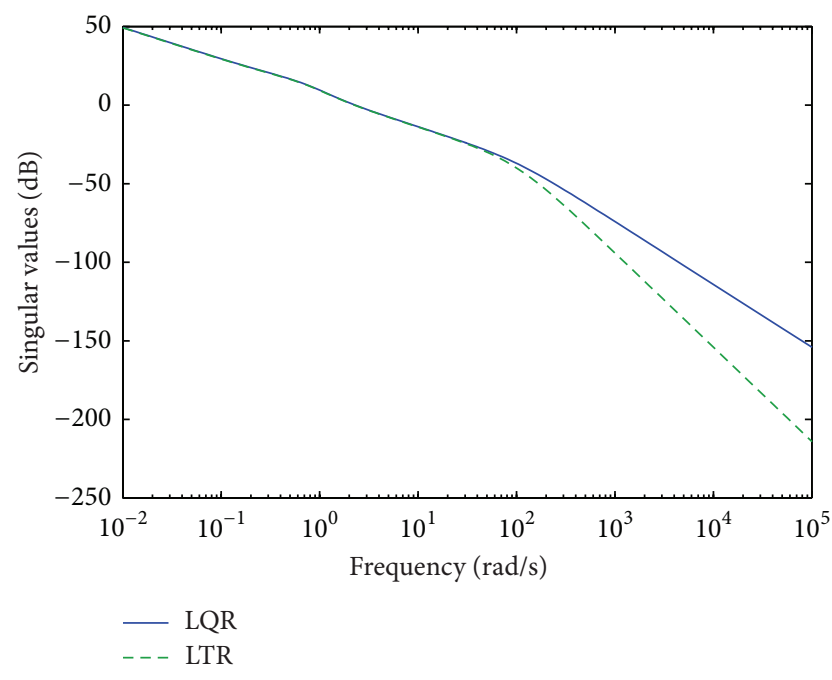

FIgURE 9: Singular values of pitch angle loop.

If we select $\rho=1 \times 10^{6}$, the singular values of pitch angle loop of $F G(s)$ denoted by LQR and $G_{c}(s) G(s)$ denoted by LTR are compared in Figure 9.

It can be seen from Figure 9 that, after the loop transfer recovery, the two singular values coincide perfectly on low frequency band, which indicates that the response of the close loop system to common control such as step input is quick and the tracking error is small even with model deviation. On high frequency band, the singular value of LTR decreases more quickly than that of LQR, which will provide excellent robustness to the high frequency disturbance such as unmodeled high frequency structural dynamics and unsteady aerodynamics. So, the LTR satisfies the required performance well.

6.3. Flight Simulation. There are 40 distributed elevons in the large scale highly flexible solar-powered UAV. The details about control actuator are omitted temporally for brief while designing the LQG/LTR controller. But in the stage of flight simulation, two kinds of actuators are employed to verify the robustness of the controller. The model of the elevons actuator is

$$
\delta_{\text {ea }}=\frac{1}{0.05 s+1} u .
$$

The model of the throttles is

$$
\delta_{t}=\frac{1}{0.5 s+1} u .
$$

Their bandwidths are 20 and $2 \mathrm{rad} / \mathrm{s}$, respectively.

Applying the LQG/LTR controller proposed above to the linear structural and flight dynamics model described in (52), when given a desired pitch angle $\theta_{c}=5^{\circ}$, the longitudinal response of solar aircraft is shown in Figures 10-14. As shown in Figure 10, the adjust time of pitch angle is about 3 seconds; because of the use of integrator, steadystate error is eliminated. Seen from Figure 11, the variation

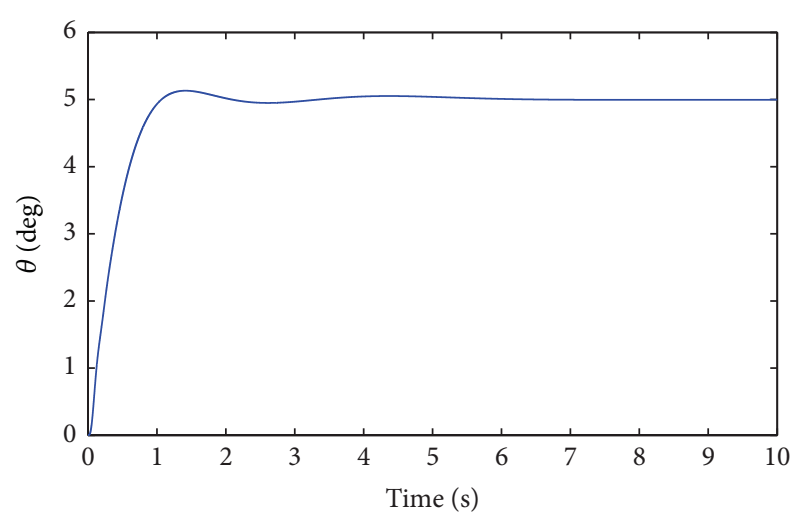

FIGURE 10: Response of pitch angle.

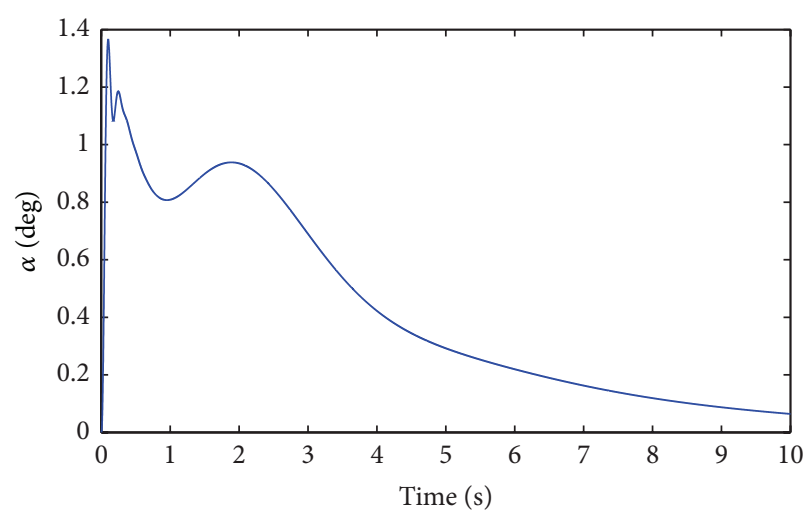

Figure 11: Response of angle of attack.

of angle of attack in the whole process does not exceed $1.4^{\circ}$, so the additional load it brings is very small, indicating that the overall movement of the highly-flexible UAV is relatively stable. Seen from Figure 12, the largest deflection angle of elevons is less than 20 degrees, and it occurs in the initial phase only, and when approaching a steady state, the deflections are no more than 5 degrees, far less than the limitation. It must be noted that the deflection angle of the 40 elevons are all different, because in addition to providing pitch angle control, distributed elevons also need to suppress the elastic deformation movement of the highlyflexible wing in the LQG/LTR controller. Seen from Figure 13, the maximum deformation of the wing occurs in the tip and the maximum magnitude of wing deformation is only about $0.09 \mathrm{~m}$, far less than the deformation of $4.58 \mathrm{~m}$ in trimming, reminding that all the elevons deflect conformably in trimming. Seen from Figure 14, the max rate of twist angle is only $0.26 \mathrm{rad} / \mathrm{s}$, corresponding to the reduced frequency of 0.0144. In a word, with distributed elevons, the use of LQG/LTR control method allows faster attitude control of the large scale highly flexible UAV while good aeroelastic deformation suppression is achieved simultaneously.

In addition, reminding that the linear structural model coincide with the nonlinear one perfectly on the condition of small deformation (shown in Figure 5). Since the LQG/LTR controller has restrained the structural deformation and 


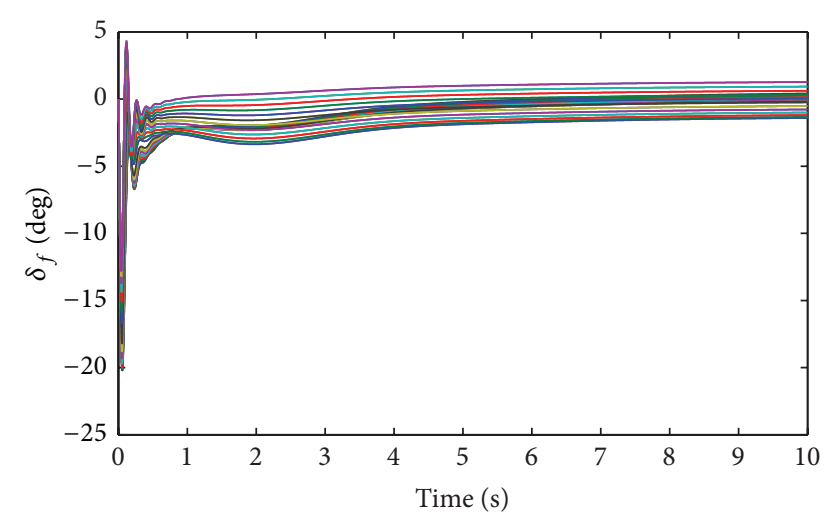

FIGURE 12: Response of elevons at different stations.

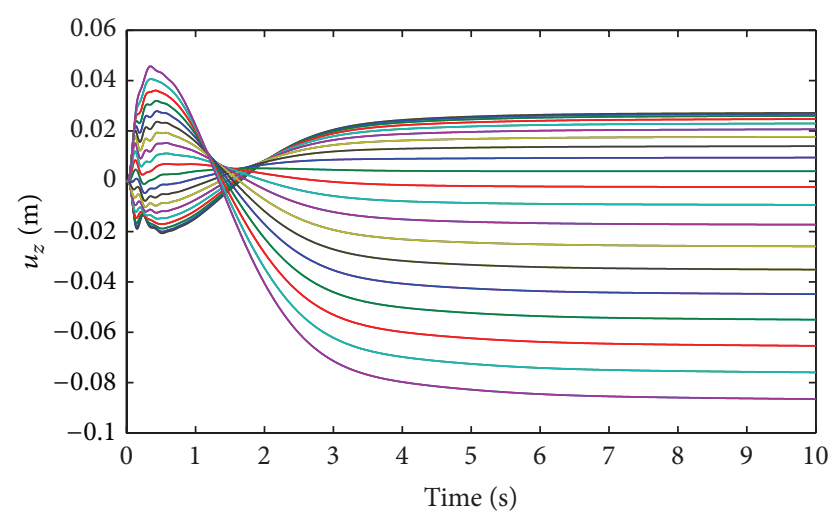

FIGURE 13: Response of vertical displacements of wing at different stations.

incident angle in a relative small range, it can be deduced that the simulation results in this section are reliable.

\section{Conclusions}

In this paper, the modeling, stability, and control characteristics of a highly flexible large scale solar-powered UAV with distributed all-span multi elevons were presented.

A geometrically nonlinear intrinsic beam model is introduced to establish the equation of motion of flexible solarpowered UAV. Based on it, the decoupled linear flight dynamics and structural dynamics equations of motions are derived in mean axis frame. Steady aerodynamics model is derived by theoretical airfoil data and corrected for 3D effect by lifting-line theory, quasisteady aerodynamics model is derived by Theodorsen unsteady aerodynamics model, and the aerodynamics derivatives of every wing sections are derived and transferred to different frames for the multirole in linear equations of flight dynamics or structural dynamics.

The nonlinear coupled structural and flight dynamics model was employed to trim the large scale flexible UAV, under the assumption that all the 40 distributed elevons deflect conformably. The maximum aeroelastic defection of wingtip is $4.58 \mathrm{~m}$ in flapwise, about $13 \%$ to the halfspan. The pitch angle at wing tip is larger than the root for about $3^{\circ}$.

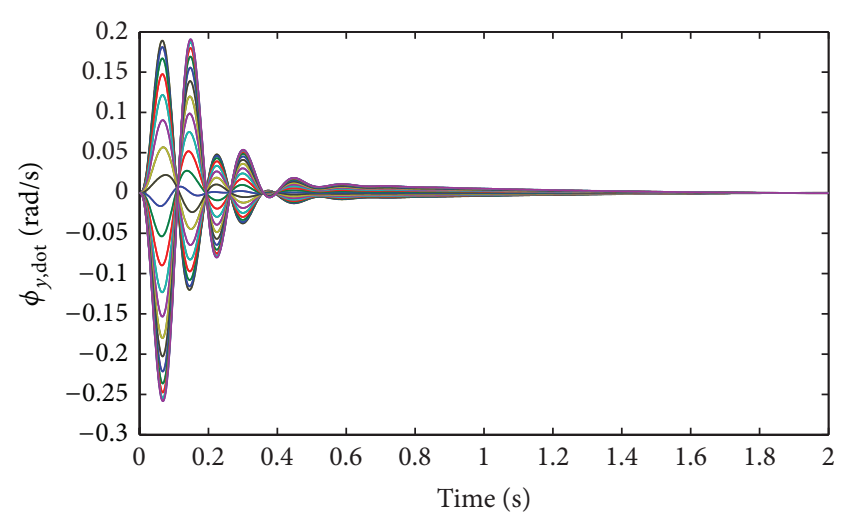

Figure 14: Response of rate of twist angle of wing at different stations.

Larger pitch angle brings larger lift and leads to larger deformation. So all the distributed elevons deflecting conformably are not the optimal control case while considering aeroelastic effects.

Three different linear dynamics models, undeformed, deformed, and elastic, were compared for longitudinal mode analysis. The results showed that the difference of three models is huge. Since the deformation of wing has increased the UAV's moment of inertia about the pitch axis, the frequency of short period mode has obviously decreased for the deformed model. The serious coupling between short period mode and 1st bending mode also significantly influences the roots of short period mode, which then results in the phugoid changing from stable to unstable. So, it is clear that the flexible model is the only one which is able to accurately estimate the longitudinal flight dynamics behaviors and control the large scale highly flexible UAV exactly.

Forty distributed all-span elevons were employed to control the attitude and suppress the aeroelastic deformation. LQG/LTR methodology was employed to accomplish the dynamics performance and robustness requirement. The flight simulation showed that the designed LQG/LTR controller can provide the adjust time of pitch angle in 3 seconds without steady-state error, and the maximum magnitude of wing deformation is only about $0.09 \mathrm{~m}$, far less than the deformation of $4.58 \mathrm{~m}$ in trimming. So, LQG/LTR control method with distributed elevons is suitable for large scale highly flexible solar-powered UAV.

\section{Nomenclature}

$P$ : Linear momentum

$H$ : Angular momentum

M: Mass or moment

I: Moment of inertia

$V$ : Linear velocity

$\Omega$ : Angular velocity

$\gamma$ : Strain

$\kappa$ : Curvature

$f$ : External force

$m$ : External moment 


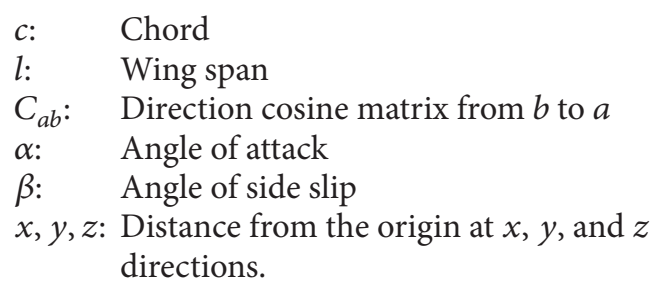

\section{Subscripts and Superscripts}

$\begin{array}{ll}g: & \text { Ground axis frame } \\ R: & \text { Deformed beam axis frame at wing root } \\ B: & \text { Deformed beam axis frame } \\ b: & \text { Undeformed beam axis frame } \\ M: & \text { Mean axis frame } \\ s: & \text { Structure axis frame } \\ a_{B}: & \text { Aero axis frame at deformed beam } \\ a_{R}: & \text { Aero axis frame at wing root } \\ a_{M}: & \text { Aero axis frame at mean axis frame } \\ \{x\}_{y}: & \text { Vector } x \text { expressed in } y \text {-frame } \\ \{x\}_{y, z}: & Z \text { part of vector } x \text { expressed in } y \text {-frame } \\ x^{\prime}: & \text { Spatial derivative of variable } x \\ \dot{x}: & \text { Temporal derivative of variable } x \\ \bar{x}: & \quad \text { Mean value over the beam element } \\ \hat{x}: & \text { Nodal value. }\end{array}$

\section{Conflict of Interests}

The authors declare that there is no conflict of interests regarding the publication of this paper.

\section{Acknowledgments}

This study is supported by the National Natural Science Foundation of China (Grant no. 11202162) and the Scientific and Technology Foundation of State Key Laboratory on UAV (Grant no. 9140C390103130C39142).

\section{References}

[1] T. E. Noll, S. D. Ishmael, B. Henwood, M. E. Perez-Davis, G. C. Tiffany, and J. Madura, "Technical findings, lessons learned, and recommendations resulting from the helios prototype vehicle mishap," Meeting Proceedings RTO-MP-AVT-145, 2007.

[2] M. J. Patil, Nonlinear aeroelastic analysis, flight dynamics, and control of a complete aircraft [Ph.D. thesis], Georgia Institute of Technology, Atlanta, Ga, USA, 1999.

[3] M. J. Patil and D. H. Hodges, "Flight dynamics of highly flexible flying wings," Journal of Aircraft, vol. 43, no. 6, pp. 1790-1798, 2006.

[4] B. Raghavan and M. J. Patil, "Flight dynamics of high aspectratio flying wings: effect of large trim deformation," AIAA 20076386, The American Institute of Aeronautics and Astronautics, 2007.

[5] W. Su and C. E. S. Cesnik, "Dynamic response of highly flexible flying wings,” AIAA Journal, vol. 49, no. 2, pp. 324-339, 2011.
[6] C. M. Shearer and C. E. S. Cesnik, "Nonlinear flight dynamics of very flexible aircraft," Journal of Aircraft, vol. 44, no. 5, pp. 1528-1545, 2007.

[7] M. J. Patil and D. H. Hodges, "Output feedback control of the nonlinear aeroelastic response of a slender wing," Journal of Guidance, Control, and Dynamics, vol. 25, no. 2, pp. 302-308, 2002.

[8] C. M. Shearer and C. E. S. Cesnik, "Trajectory control for very flexible aircraft," AIAA 2006-6316, 2006.

[9] B. Raghavan and M. J. Patil, "Flight control for flexible, highaspect-ratio flying wings," Journal of Guidance, Control, and Dynamics, vol. 33, no. 1, pp. 64-74, 2010.

[10] X. Li and R. K. Agarwal, "Application of eeduced-order-models to robust control of the dynamics of a flexible aircraft," AIAA 2003-5504, 2003.

[11] M. Athans, "A tutorial on LQG/LTR method," in Proceedings of the American Control Conference, Seattle, Wash, USA, June 1986.

[12] T. E. Gibson, A. M. Annaswamy, and E. Lavretsky, "Modeling for control of very flexible aircraft," in Proceedings of the AIAA Guidance, Navigation, and Control Conference, AIAA 2011-6202, Portland, Ore, USA, August 2011.

[13] D. H. Hodges, "A mixed variational formulation based on exact intrinsic equations for dynamics of moving beams," International Journal of Solids and Structures, vol. 26, no. 11, pp. 1253-1273, 1990.

[14] R. Palacios, J. Murua, and R. Cook, "Structural and aerodynamic models in nonlinear flight dynamics of very flexible aircraft," AIAA Journal, vol. 48, no. 11, pp. 2648-2659, 2010.

[15] S. S. Rao, Mechanical Vibrations, Pearson Education, Upper Saddle River, NJ, USA, 4th edition, 2004.

[16] B. Raghavan, Flight dynamics and control of highly flexible flyingwing [Ph.D. thesis], Virginia Polytechnic Institute and State University, 2009.

[17] D. H. Hodges, "Geometrically exact, intrinsic theory for dynamics of curved and twisted anisotropic beams," AIAA Journal, vol. 41, no. 6, pp. 1131-1137, 2003.

[18] D. A. Peters and W.-M. Cao, "Finite state induced flow models part I: two-dimensional thin airfoil," Journal of Aircraft, vol. 32, no. 2, pp. 313-322, 1995.

[19] J. D. Anderson, Fundamentals of Aerodynamics, McGraw-Hill, 4th edition, 2007.

[20] Z. Fang, W. Chen, and S. Zhang, Aeronautic Vehicle Flight Dynamics, Beihang University Press, 1st edition, 2005 (Chinese).

[21] R. Wang, "Research on dynamics and control of highly flexible solar-powered UAV," Postdoctoral Report, Northwestern Polytechnology University, Xi'an, China, 2011 (Chinese). 


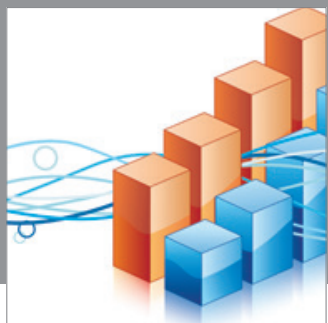

Advances in

Operations Research

mansans

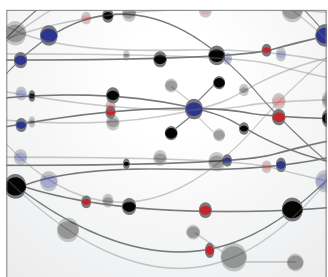

The Scientific World Journal
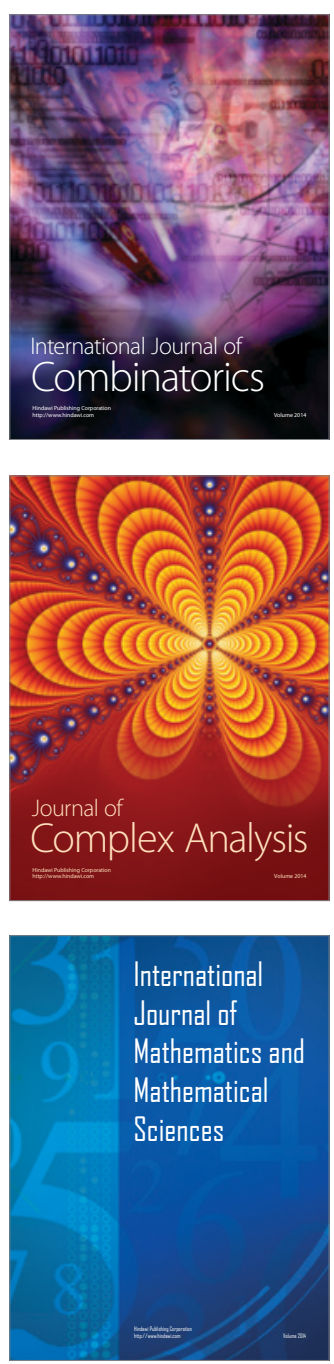
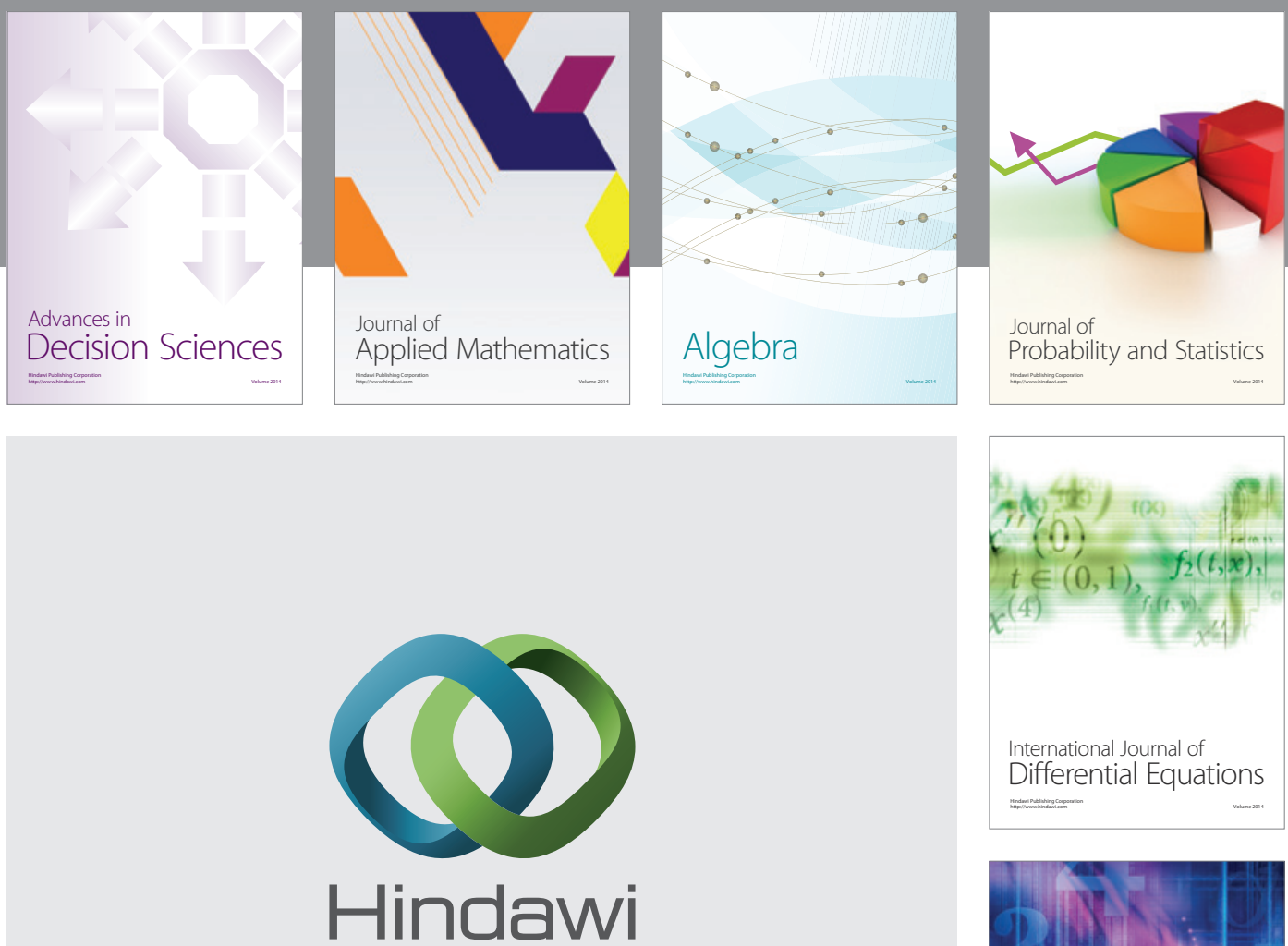

Submit your manuscripts at http://www.hindawi.com
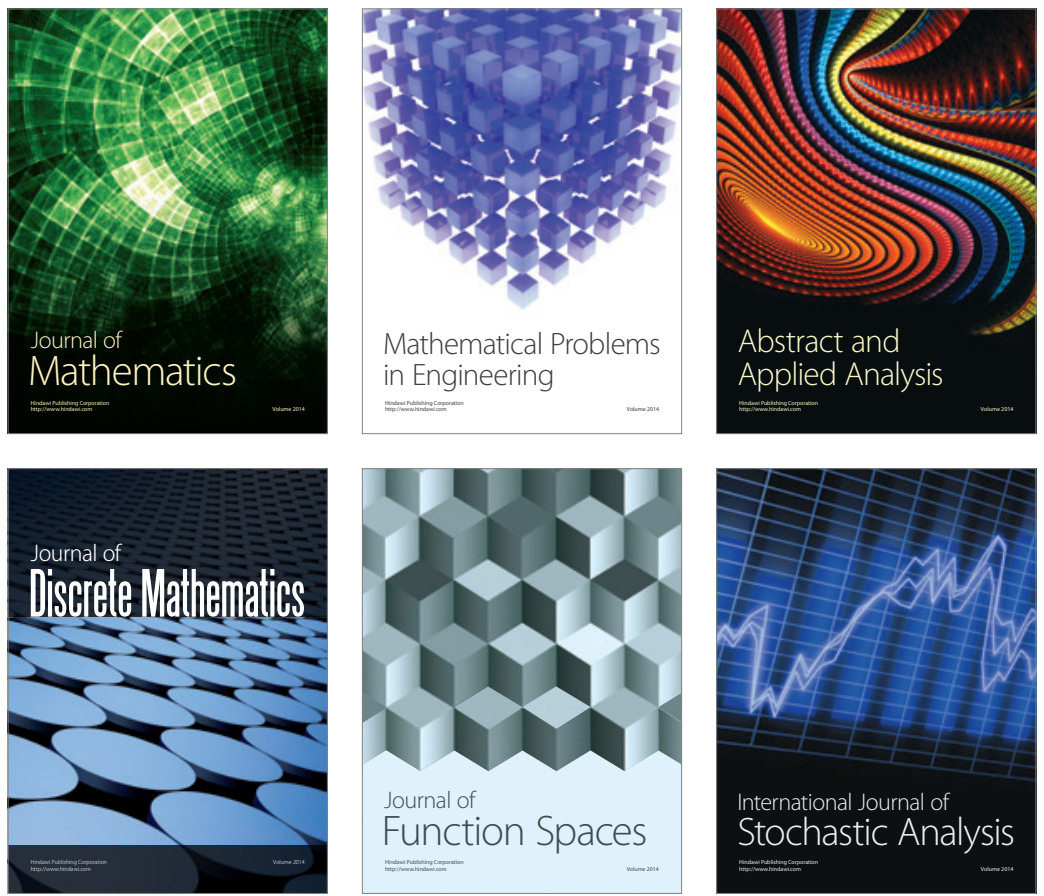

Journal of

Function Spaces

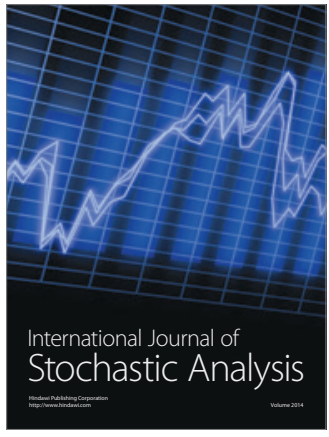

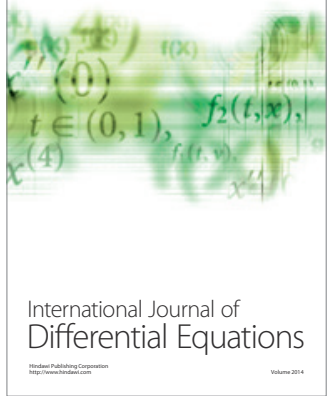
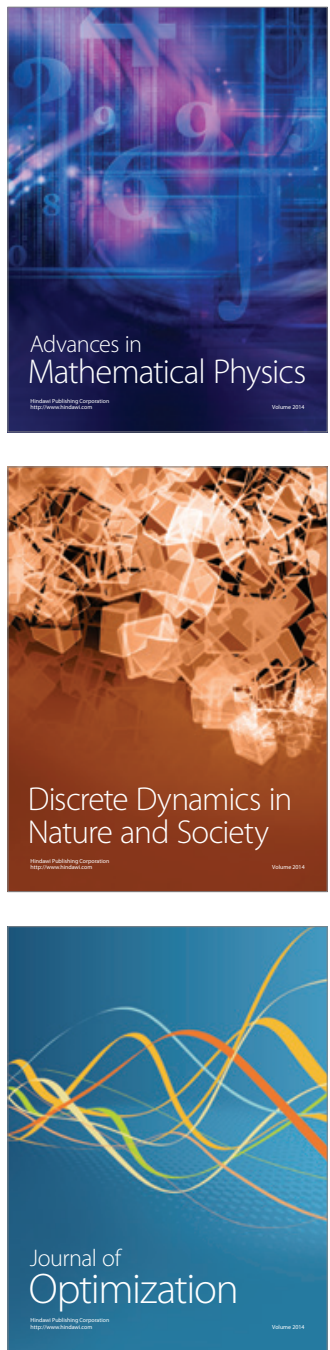